\title{
Efficient Method for Offline Kannada and English Handwritten Word Recognition
}

\author{
Dayanand S. Patil ${ }^{1}$, Anil S.Naik ${ }^{2}$ \\ Dept. of CSE, Walchand Institute of Technology, Solapur, India ${ }^{1}$ \\ Dept. of IT, Walchand Institute of Technology, Solapur, India ${ }^{2}$
}

\begin{abstract}
Recognition of handwritten text has been one of the active and challenging areas of research in the field of image processing and pattern recognition. It has numerous applications which include postal mail application, reading aid for blind and conversion of any handwritten document into electronic form. In this paper we focus on recognition of Karnataka district names in Kannada and 20 different English words from a given scanned word image with the help of classifiers. The first step is image acquisition which acquires the scanned image followed by noise filtering, smoothing and resizing of scanned image. Feature Extraction improves recognition rate and misclassification. We use edge detection algorithm to extract sharp edges and features are extracted to train the classifier to classify and recognize the handwritten word.
\end{abstract}

Keywords: Handwritten word recognition, feature extraction, Dominant Points, Edge Detection algorithm and Classification (key words).

\section{INTRODUCTION}

As the world moves closer to the concept of the "paperless This method has been efficiently used in speech office," more and more communication and storage of processing, sign language analysis, online and offline documents is performed digitally. Documents and files character recognition etc.

that were once stored physically on paper are now being English is most popular language and using in almost converted into electronic form in order to facilitate quicker offices. English Script has 26 Characters, Comprising 5 additions, searches, and modifications, as well as to vowels and 21 consonants. Kannada is the official prolong the life of such records. A great portion of language of the South Indian state of Karnataka. It has its business documents and communication, however, still own script derived from Bramhi script. Kannada script has takes place in physical form i.e. handwritten and the fax a base set of 49 characters, comprising 15 vowels and 34 machine remains a vital tool of communication consonants. Further there are distinct symbols that modify worldwide. Because of this, there is a great demand for the base consonants, called consonant and vowel modifiers software, which automatically extracts, analyzes, and stores information from physical documents for later retrieval. The overwhelming volume of paper-based data in corporations and offices challenges their ability to manage documents and records. Computers, working faster and more efficiently than human operators, can be used to perform many of the tasks required for efficient document and content management. Computers understand alphanumeric characters as ASCII code typed on a keyboard where each character or letter represents a recognizable code. However, computers cannot distinguish characters and words from scanned images of paper documents. Therefore, where alphanumeric information must be retrieved from scanned images such as commercial or government documents, tax returns, passport applications and credit card applications, characters must first be converted to their ASCII equivalents before they can be recognized as readable text. Euclidean metric is a popular method to define similarity and index time series, but it is very brittle in computing similarity between time series with different time phases, DTW distance can overcome this problem by searching an optimal match between two given time series. DTW uses a dynamic programming technique to find the minimal distance between two time series, where sequences are warped by stretching or shrinking the time dimension. [2]. The number of these modifiers is the same as that of the base characters. The characters called aksharas are formed by graphically combining the symbols corresponding to consonants, consonant modifiers (optional) and vowel modifiers using well defined rules of combination.

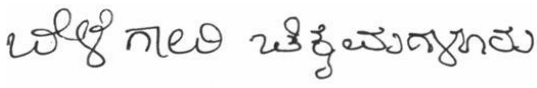

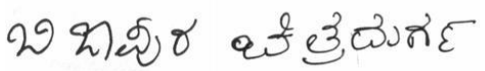

$$
\begin{aligned}
& \text { जowent? }
\end{aligned}
$$

Fig. 1. Sample dataset of district names of Karnataka
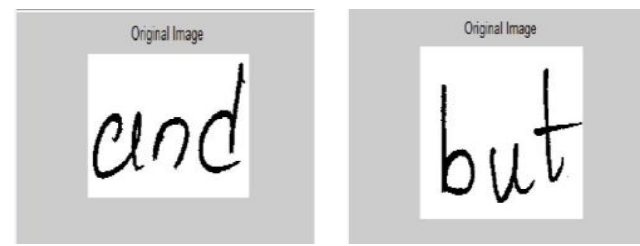

Fig. 2. Sample dataset of English word

Though so many research papers are available in the literature, still handwritten word recognition is an open problem, especially in the domain of feature design and classification methodology. Researchers have attempted to 
address this problem from different research perspectives. Most of such attempts are not comparable due to, 1) non availability of standard data sets and, 2) variations in research objectives.

\section{PROPOSED METHOD}

In this proposed methods two different feature extraction methods are used. And both methods are working for both Kannada and English languages separately. A total of 1200 Kannada and 1200 English words from around 60 people were obtained and stored as data set. Handwritten characters usually come in various sizes, shapes and fonts and writers were chosen from schools, colleges and Home. The database was restricted to district names of Karnataka and 20 different English words. In this paper two different feature extraction methods are used.

There are 4 Stages in the word recognition process:

1. Data collection and Pre-processing

2. Feature extraction

3. Classification

4. Recognition

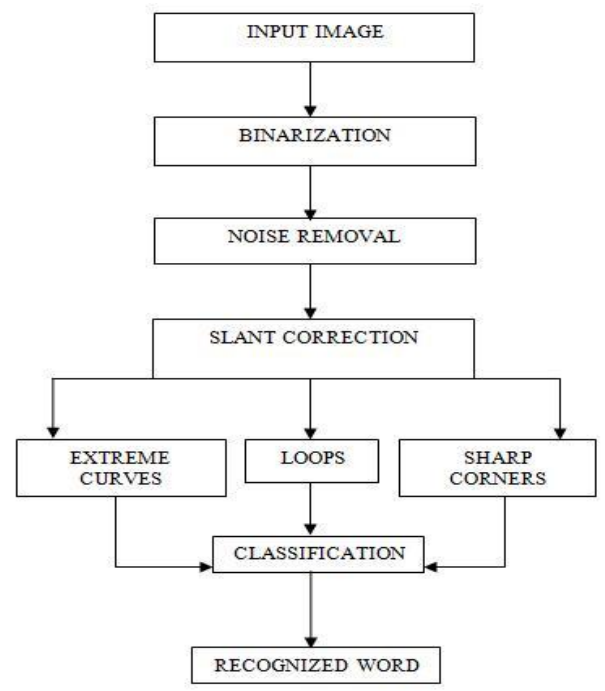

Fig. 3. Proposed mode

A. Binarazation

A handwritten document is first scanned and is converted into a gray scale image [1]. Binarization is a technique by which the gray scale images are converted to binary images. The goal is to remove only the background, by setting it to white, and leave the foreground image unchanged. Thus, binarization separates the foreground and background information.

B. Noise Removel

Scanned documents often contain noise that arises due to printer, scanner, print quality, age of the document, etc. Therefore, it is necessary to filter this noise before we process the image.

Median filters are particularly effective in the presence of impulse noise, also called 'salt - and - pepper' noise because of its appearance as white and black dots superimposed on an image. For every pixel, a $3 \times 3$ neighborhood with the pixel as center is considered [1]. In median filtering, the value of the pixel is replaced by the median of the pixel values in the $3 \times 3$ neighborhood.
C. Skew Detection and Correction.

When a document is fed to the scanner either mechanically or by a human operator, a few degrees of tilt is unavoidable. Skew angle is the angle that the lines of text in the digital image make with the horizontal direction [1]. D. Thinning.

The purpose of thinning is to reduce the image components to their essential information so that further analysis and recognition are facilitated. For instance, an alphabet can be handwritten with different pens giving different stroke thicknesses, but the information presented is the same [1]. This enables easier subsequent detection of pertinent features

E. Edge detection.

Canny edge detection algorithm:

Step 1: Read an input image where the sharp edges need to be detected.

Step 2: Call BW=edge(Input_image,'canny',thresh) within Matlab.

Step 3: The above function takes three arguments i.e., 'Input image', 'canny' and 'thresh'.

Step 4: Set the 'thresh' value is a two-element vector in which the first element is the low threshold, and the second element is the high threshold. If we specify a value for threshold, this value is used for the high threshold and 0.4* thresh is used for the low threshold. If we do not specify threshold value or if thresh is empty, edge chooses low and high values automatically.

Step 5: If any edge response is above a high threshold, those pixels constitute definite output of the edge detector. Step 6: Individual weak responses usually correspond to noise, but if these points are connected to any of the pixels with strong responses then there are more chances to be actual edges in the image. Such connected pixels are treated as edge pixels if their response is above a low threshold.

F. Feature Extraction.

Major goal of feature extraction is to extract a set of features which maximizes the recognition rate with least amount of elements.

A large number of feature extraction methods are reported in literature; but the methods selected depend on the given application. Since our project is specific to postal application to identify the districts of Karnataka and 15 classes of English words the features we considered are: Corner points, curves and loops.

We used two different feature extraction methods and both methods are working for both Kannada and English Languages.

METHOD I:

Steps to detect corner points:

Step 1: Read the input image, it could be gray or binary image.

Step 2: Detect edges using canny edge detector

Step 3: Extract curves from binary edge map

Step 4: Calculate the curvature and find the curvature local maxima as corner points

Step 5: Compare each maxima with its two local minima to remove false corners

Step 6: Finally we get actual corner points. 
Freeman's chain code (FCC) is one of the techniques representations based on the boundary extraction which useful for image processing, shape analysis and pattern recognition[7]. Chain code representation gives a boundary of character image where the codes represent the direction of where is the location of the next pixel.
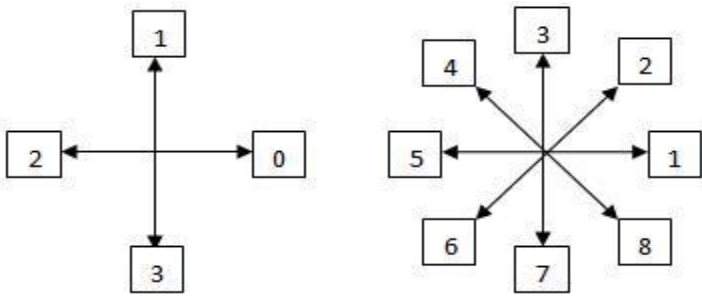

Fig.6: Two Directions of FCC:
(a) 4-Neighborhood
(b) 8-Neighborhood.

Freeman's chain code algorithm:

Step 1: Create a binary image from the original input image.

Step 2: Save the image in matrix of 0's for background and 1's for the object. So, while this process is in progress, we compute the area of the object by adding for each iteration.

Step 3: Compute chain code by scanning the image to find the starting pixel of the object. From that pixel, we traverse the boundary and decide directions and save them as an array or list. This step is repeated until we reach the end pixel [5].
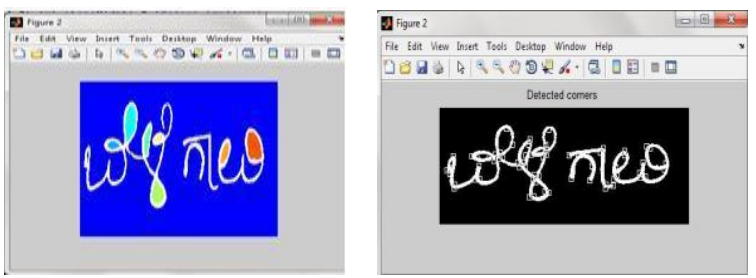

(a) Loops and (b) Corner points in a word

Fig.7: Features extracted

\section{METHOD II}

In this method feature extraction done based on Dominant Points and Extreme Curvature. Dominant points in shapes [8] refer to points in one of the following sets:

- End points of the shape (that is, points simply connected).

- Points corresponding to local extremes of curvature.

- Intersection points.

These points are used to describe sudden changes in the lines of the cursive handwritten word.

The features are extracted from thinned word based on dominant points in the word. This method calculates the distance of each connected (by dominant point) edge and also calculates the orientation of each edge with respect to $\mathrm{x}$-axis.

Steps to detect Dominant points:

Step 1: Pick 3X3 window and slide the window over the image.

Step 2: If we find white pixel at center check the fallowing condition.
If $\mathrm{Cn}=3$ or $\mathrm{Cn}=1$ or $\mathrm{Cn}=4$; take it as dominant point . (connectivity number $C_{n}$ shows in figure 4 )

Figure 8: The connectivity number $C_{n}$. Steps to detect Extreme Curvature
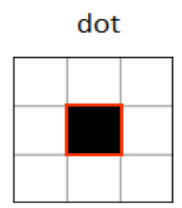

$C_{n}=0$

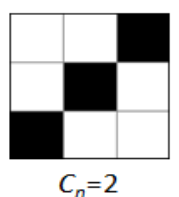

end-point

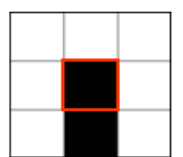

$C_{n}=1$

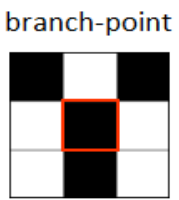

$C_{n}=3$

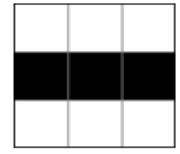

$C_{n}=2$

cross-point

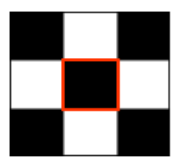

$C_{n}=4$
Steps to detect Extreme Curvature points:

Step 1: Pick $3 X 3$ window and slide over the image; If we get unprocessed white pixel then walk through the neighborhood white pixels.

Step 2: While moving find slope $(\mathrm{dy} / \mathrm{dx}=(\mathrm{y} 1-\mathrm{y} 2) /(\mathrm{x} 1-\mathrm{x} 2))$. Step 3: Compare current slope value with previous value; if we get change in slope and take previous pixel as dominant point and move to next pixel.

Step 4: If we get end point again we will scan the image to get unprocessed white pixel.

(Repeat step 1 to 4 until we process all the white pixels in the image)
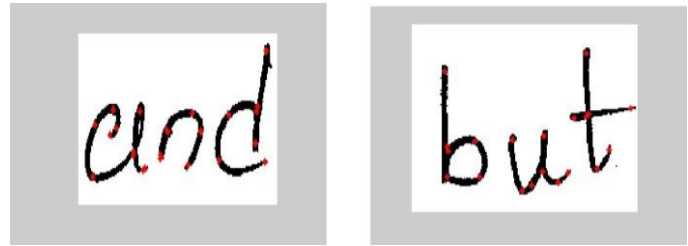

Fig 9: Detected Domonant Points and Extreme Curvature

\section{CLASSIFICATION}

Classification stage in an HWR (Handwritten Word Recognition) process assigns labels to word images based on the features extracted and the relationships among the features. The principal approaches to decision-theoretic recognition are: minimum-distance classifiers, statistical classifiers and neural networks. There are different types of minimum-distance classifiers like Manhattans, Euclidean distance etc. The Euclidean distance measure is the "standard" distance measure between two vectors in feature space.DTW offers a more flexible way to compensate for the variations like inter character and intra character spacing than linear scaling. Here we use the Euclidean distance and DTW algorithm to recognize the word images based on the features extracted using minimum difference value.

\section{RESULT}

The database is created by collecting data from 60 different writers so that recognition engine could be trained with different styles of handwriting. As we were 
specific to limited words we collected writings of all the 30 district names of Karnataka and 20 different English words from each writer. So a total of 1200 Kannada words and 1200 English words dataset were created. The proposed method was trained with a dataset of 250 bitmap images digitized at $300 \mathrm{dpi}$ and the recognition engine is tested for the accuracy. Euclidean distance classifier is used to classify the test images and from experiments done $92 \%$ of recognition rate is obtained from both methods. Advantage is both individual methods are works for both languages.

\section{CONCLUSION}

The handwritten word is a widely varying recognition target. Relatively high computational power and large storage are required to build a successful HWR system. In this project a method for off-line handwritten word recognition is implemented. This method is based on features identification and distance measure. Comparison of results with other researchers is difficult because of differences in experimental details, the actual handwriting used and the method of data collection.. Euclidean distance function is used to classify the images, which gives the class id of the matched image based on minimum distance value.

The HWR system for Kannada and English is explored and this method works for connected handwritings with improved recognition rate. The recognition rate can be optimized by using minimum number of features in training process.

\section{REFERENCES}

[1] Ahmed Sahlol and Cheng Suen, "A Novel Method for the Recognition of Isolated Handwritten Arabic Characters", Department of Computer Teacher preparation, Damietta University, Damietta, Egypt and Department of Computer Science, Concordia University, Canada, 2013.

[2] Rituraj Kunwar, K.Shashikiran and A.G.Ramakrishnan, "Online Handwritten Kannada Word Recognizer with Unrestricted Vocabulary", Dept of Electrical Engineering, IISc, Bangalore, India,2010.

[3] Venkat Rasagna, Anand Kumar, C.V.Jawahar and R.Manmatha, "Robust Recognition of Documents by Fusing Results of Word Clusters", Center for Visual Information Technology, IIIT, Hyderabad, India,2003.

[4] Toni M. Rath and R. Manmatha, "Word Image Matching Using Dynamic Time Warping", Multi-Media Indexing and Retrieval Group Center for Intelligent Information Retrieval University of Massachusetts Amherst, MA 01003.

[5] Dr. Azzam Talal Sleit and Rahmeh Omar Jabay, "A Chain Code Approach for Recognizing Basic Shapes", Information Technology College University Of Jordan, Amman, Jordan,2006.

[6] Ankush Acharyya, Sandip Rakshit, Ram Sarkar, Subhadip Basu and Mita Nasipuri, "Handwritten Word Recognition Using MLP Based Classifier", IJCSI International Journal of Computer Science Issues, Vol. 10, Issue 2, No 2, March 2013.

[7] Dewi Nasien, Habibollah Haron and Siti Sophiayati Yuhaniz, "The Heuristic Extraction Algorithms for Freeman Chain Code of Handwritten Character", Faculty of Computer Science and Information System (FSKSM) Universiti Teknologi Malaysia Skudai, 81310, Malaysia

[8] J. R. Rico, 'Off-line Cursive Handwritten Word Recognition Based On Tree Extraction and Optimized Classification Distance “, E03071 Alicante. SPAIN. 